\title{
Performance Evaluation of Scheduling Algorithms for 4G (LTE)
}

\author{
Bamidele Moses Kuboye \\ Department of Computer Science, Federal University of Technology, Akure, Nigeria \\ Email: bmkuboye@futa.edu.ng, kubonline@yahoo.co.uk
}

How to cite this paper: Kuboye, B.M. (2018) Performance Evaluation of Scheduling Algorithms for 4G (LTE). Communications and Network, 10, 152-163. https://doi.org/10.4236/cn.2018.104013

Received: July 10, 2018

Accepted: October 27, 2018

Published: October 30, 2018

Copyright $\odot 2018$ by author and Scientific Research Publishing Inc. This work is licensed under the Creative Commons Attribution International License (CC BY 4.0).

http://creativecommons.org/licenses/by/4.0/

\section{(c) (i) Open Access}

\begin{abstract}
Long Term Evolution (LTE) is designed to revolutionize mobile broadband technology with key considerations of higher data rate, improved power efficiency, low latency and better quality of service. This work analyzes the impact of resource scheduling algorithms on the performance of LTE (4G) and WCDMA (3G) networks. In this paper, a full illustration of LTE system is given together with different scheduling algorithms. Thereafter, 3G WCDMA and 4G LTE networks were simulated using Simulink simulator embedded in MATLAB and performance evaluations were carried out. The performance metrics used for the evaluations are average system throughput, packet delay, latency and allocation of fairness using Round Robin, Best CQI and Proportional fair Packet Scheduling Algorithms. The results of the evaluations on both networks were analysed. The results showed that $4 \mathrm{G}$ LTE network performs better than $3 \mathrm{G}$ WCDMA network in all the three scheduling algorithms used.
\end{abstract}

\section{Keywords}

Long Term Evolution, Third Generation (3G), Fourth Generation (4G), Network, Algorithms, Scheduling

\section{Introduction}

A lot of people across the nation have adopted mobile broadband as their primary means of accessing Internet, voice communication, entertainment services and text messages. Therefore, the demand for mobile broadband services increases every time. According to [1], Mobile broadband subscriptions reached over 1.4 billion in 2012. The International Telecommunications Union (ITU) has said the population of mobile broadband users across the world was expected to hit 4.3 billion at the end of 2017 from 3.2 billion it was in 2015 [2] [3]. 
In 2018, the number of LTE subscriptions is forecast to reach 3.3 billion [3]. Explosive growth in wireless subscribers, increasing deployment of intensive multimedia applications for mobile platforms like content downloading, video streaming, gaming, reliable communication and demand for satisfactory user experience are driving the driving force for good quality of service (QoS) in mobile broadband network. LTE provides high speed and high mobile broadband data capacity at a lower cost per bit, low latency and improved customer experience [4].

Prior to the advent of LTE, wireless technology generationshas been delivering functionalities like voice, short message service (SMS), video calls and data file transfers. The first generation wireless technology (1G) was successful in moving the basic mobile voice, while the second generation $(2 \mathrm{G})$ introduced higher capacity and coverage than the $1 \mathrm{G}$. Thereafter, $2.5 \mathrm{G}$ was introduced to pave way for the data connection on cellular network, thus, revolutionize the adoption of data connection on the cellular network till date [5]. Third generation (3G) Mobile Technology was created to support Internet connectivity and packet-switched services as well as circuit switched services. International mobile telecommunication (IMT)-2000 a standard that developed 3G communications system chooses data rates aims to realize $144 \mathrm{Kbps}, 384 \mathrm{Kbps}$, and $2 \mathrm{Mbps}$ under high mobility, low mobility, and stationary environments, respectively [6]. High-Speed Packet Access (HSPA) is a collection of protocols that extend and improve the performance of $3 \mathrm{G}$ mobile protocols. It has High-Speed Downlink Packet Access (HSDPA) and High-Speed Uplink Packet Access (HSUPA) standards. HSDPA deployment supports 1.8 Mbps, 3.6 Mbps, 7.2 Mbps and 14.4 Mbps in downlink data applications, with shorter connection and response times while HSUPA support the up-link speeds up to $5.76 \mathrm{Mbps}$ [7]. The third generation (3G) brought the quest for data at higher speeds to open the gates for mobile broadband experience which was further realized by the fourth generation (4G).

The Fourth generation (4G) provides access to wide range of telecommunication services [8]. $4 \mathrm{G}$ allows the integration of available heterogeneous and homogeneous networks into a single platform capable of supporting user roaming between them while not interrupting active communications. The $4 \mathrm{G}$ is driven by low cost, high-speed data, application ubiquity, high degree of personalization and synchronization between various user appliances [9]. 3G is based on two parallel infrastructures consisting of circuit switched and packet switched network nodes respectively, while $4 \mathrm{G}$ is based on packet switching only. It consists of end-to-end IP solution with better quality data, video and sound services due to high bandwidth, and convergence of networks services such as enterprise, fixed and cellular [7]. The design target for radio performance is to achieve a scalable capacity from $100 \mathrm{Mbps}$ high speeds and above compared to the $3 \mathrm{G}$ best performance of 14.4 Mbps using HSDPA and other 3G technologies.

The Long Term Evolution (LTE) belongs to the family of $4 \mathrm{G}$ or next generation wireless communication $[10]$. $4 \mathrm{G}$ wireless technology allow an immediate 
access to services that offer information on demand at a very high data communication speed because it was designed to provide 3 times faster speed than $3 \mathrm{G}$. It has higher data rates of about $300 \mathrm{Mbps}$ peak downlink and $75 \mathrm{Mbps}$ peak uplink. LTE supports scalable carrier bandwidths from $1.4 \mathrm{MHz}$ to $20 \mathrm{MHz}$ and supports both Frequency Division Duplex (FDD) and Time Division Duplex (TDD); thus resulted to better performance than 3G. LTE gives QoS to agencies of all sizes and people of all races including those in remote rural areas without wireless internet coverage. LTE supporting technology includes OFDM which can achieve the targeted high data rates with simpler implementation and relatively low cost and power efficient hardware [11]. It removes the limitations of previous wireless technology by deploying OFDMA for downlink and SC-FDMA technology for uplink. SC-FDMA is technically similar to OFDMA but it suits better for hand-held devices because it is less demanding in battery power. LTE uses MIMO technology to send data, thereby, minimizing noise effect, increase throughput, and spectrum utilization. The basic idea of MIMO is to use multiple smart antennas at the receiver end and use multiple transmitters when sending the data. LTE is the technological path followed to achieve $4 \mathrm{G}$ network speeds.

\section{LTE Network Architecture}

LTE aarchitecture is a simple flat IP based that reduces latency and it is compatible with third generation partnership project (3GPP) and non 3GPP Technologies. The LTE network architecture comprised of User Equipment (UE), Evolved UMTS Terrestrial Radio Access Network (E-UTRAN) and Evolved Packet Core (EPC). The LTE uses Uu interface to connect the UE with the eNB while S1 interface connects the eNB to EPC. Also, X2 interface connects eNB together as illustrated in Figure 1 [12].

\subsection{The User Equipment (UE)}

User Equipment (UE) is the device used by the end user for communication. This is usually a mobile device such as smart phone or a data card that could be

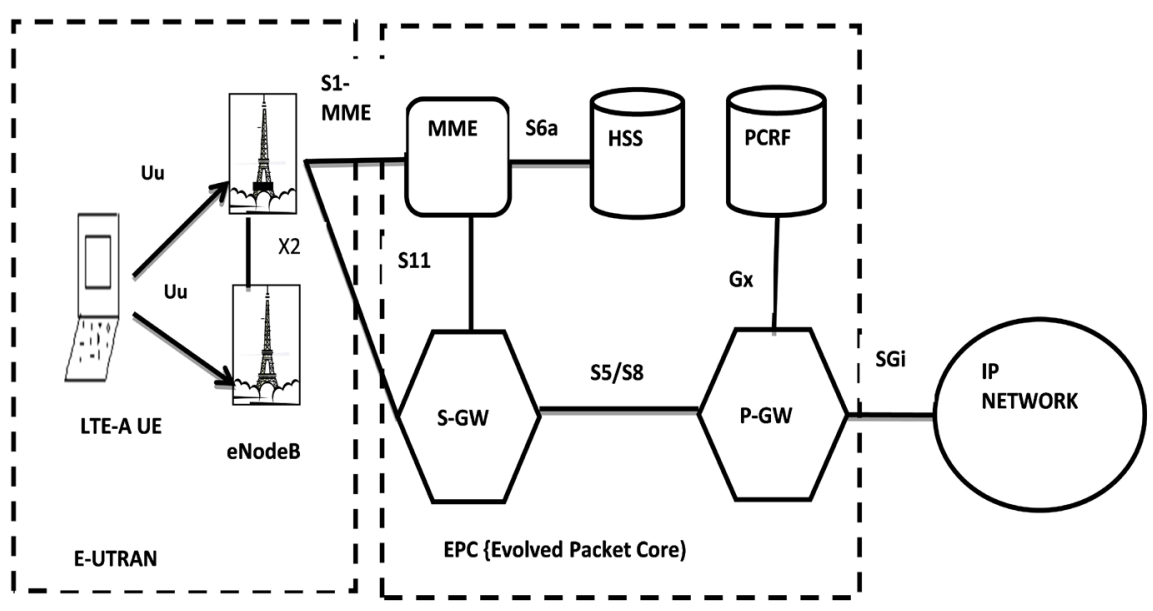

Figure 1. LTE Architecture [13]. 
attached to a computer. It provides measurements that indicate channel conditions to the network. The UE has Universal Subscriber Identity Module (USIM) that stores user-specific data similar to SIM card used nin the prevoius cellular Networks.It helps keep subscriber's information like user's phone number, home network identity and security keys.

\subsection{The Evolvned UMTS Terrestrial Radio Access Network (E-UTRAN)}

The E-UTRAN handles the radio communications between the Users Equipment and the evolved packet core. It has the evolved base station, known as eNodeB or eNB [14]. Each eNB is a base station that controls the mobiles in one or more cells [12]. Several eNodeB are connected together using the X2 interface which is designed to minimize packet loss caused by mobility of UE. The LTE-Uu interface is the standard that connects the UE to eNodeB, and it enables transmission or reception and radio resource management functions. LTE eNodeB uses Orthogonal Frequency Division Multiplexing (OFDM) for the downlink and single carrier frequency division multiple access (SC-FDMA) for the uplink and employs Multiple Input Multiple Output (MIMO) with up to four antennas per station.

\subsection{The Evolved Packet Core (EPC)}

The EPC communicates with external packet data networks such as the internet, private corporate networks or the IP multimedia subsystem. The architecture of EPC can be grouped into two main planes: the user plane and the control plane. Mobility management entity (MME) is the core of the control plane while serving gateway (S-GW) forms the core of the user plane. The S1 interface connects the eNodeB to the MME and S-GW.

\subsection{Resource Block and Bandwidth Configurations}

One resource block has 12 OFDM subcarriers, $180 \mathrm{KHz}$ bandwidth, $15 \mathrm{KHz}$ subcarrier spacing, timeslot of $0.5 \mathrm{~ms}$ and 7 OFDM symbols. LTE resource block (RB) is the smallest resource element assigned by the scheduler. RB consists of 72 (12 Subcarriers $\times 6$ OFDM) or 84 (12 Subcarriers $\times 7$ OFDM) resource elements REs. Scheduler function is to distribute resources (time and frequency) among users for each Transmission Time Interval (TTI) of $1 \mathrm{~ms}$ [15]. The number of RBs in a resource grid depends on the size of the bandwidth. The LTE operates in the bandwidth of $1.4 \mathrm{MHz}$ up to $20 \mathrm{MHz}$, with number of radio blocks (RBs) ranging from 6 to 100 for bandwidths $1.4 \mathrm{MHz}$ to $20 \mathrm{MHz}$ respectively, as shown in Table 1.

\subsection{LTE Framestructure}

LTE radio downlink transmission frame has duration of $10 \mathrm{~ms}$. Frame is divided into $10 \mathrm{sub}$-frames of $1 \mathrm{~ms}$ each. Furthermore, each sub-frame is divided into 2 time slots of $0.5 \mathrm{~ms}$ duration as shown in Figure 2. Each time slot consists of 6 
or 7 Orthogonal Frequency Division Multiplexing OFDM symbols [15].

\section{Scheduling Algorithms}

\subsection{Round Robin (RR) Scheduler}

Round robin algorithm uses a principle of sharing resources on an equal time slots basis and does not consider the channel quality information from participating user equipment [17]. Each active UE in a cell have equal access to resources and services at equal amount of timeslots. This means that the same amount of radio resources is given to each communication link but it might not achieve a fair results in the sense of providing the same service quality to all communication links because the users are not transmitting the same data type or belong to the same traffic class.

\subsection{Best CQI}

This scheduler scheme assigns RBs to the UE with the best channel conditions. To perform scheduling, UE generates the channel quality indicator (CQI) information and it feeds them back to the eNodeB periodically in quantized form

Table 1. Bandwidth configuration and resource block allocation [16].

\begin{tabular}{ccc}
\hline $\begin{array}{c}\text { Channel } \\
\text { Bandwidth } \\
(\mathrm{MHz})\end{array}$ & $\begin{array}{c}\text { Number of resource blocks } \\
\text { (Transmission configuration bandwidth) }\end{array}$ & $\begin{array}{c}\text { Maximum } \\
\text { occupied Bandwidth }\end{array}$ \\
\hline 1.4 & 6 & 1.08 \\
3 & 15 & 2.7 \\
5 & 25 & 4.5 \\
10 & 50 & 9.0 \\
15 & 75 & 13.5 \\
20 & 100 & 18.0 \\
\hline
\end{tabular}

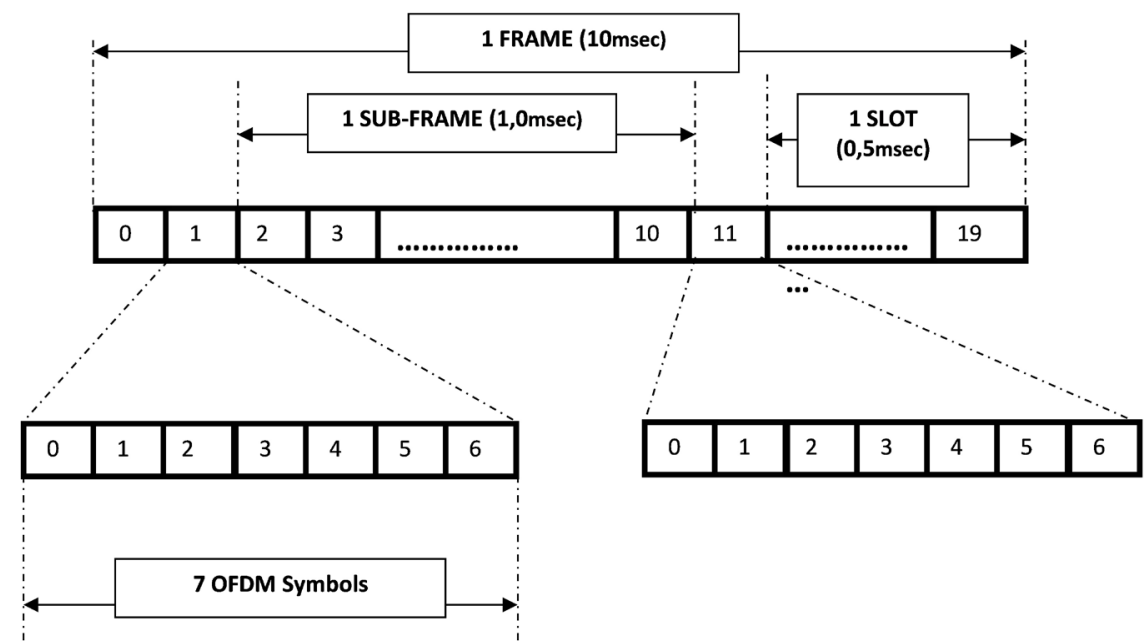

Figure 2. LTE frame Structure [15]. 
but with a certain delay. These CQI information contain the value of the signal-to-noise-plus-interference-ratio (SINR) measured by the UE. A higher value of CQI indicates a better channel condition [18]. The best CQI is selected for scheduling based on the CQI received. Best CQI scheduling scheme can increase cell throughput at the expense of worst fairness. In this scheduling mechanism, UEs located far from the base station are likely to be starved and not scheduled.

\subsection{Proportional Fair Scheduler}

Proportional fair scheduler is a compromise between RR and Maximum Best CQI. Its main targets are to provide maximum rate and to prevent UE starvation. A priority function is used to position the UEs and thereafter, the UE with the highest priority is assigned resources [18]. This scheduling algorithm assigns the RBs to the UE with the best relative channel quality. There are various versions of PF scheduling based on values that it takes into account. The main goal of this scheduling algorithm is to achieve a balance between maximizing the cell throughput and fairness by allowing all users achieve a minimum QoS.

\section{Related Work}

A comparison of $3 \mathrm{G}$ and $4 \mathrm{G}$ network in order to list out the drawback and merits of the two evolution of wireless Technology was done by Kumar and Suman [19]. The research focuses on their Architecture, speed, supporting technology, bandwidth and QoS. They gave full description of LTE and 3G Architecture stating that LTE Architecture is flat IP based architecture and made it a better choice than 3G. LTE because it reduces latency and cost and its infrastructures consist of a set of various networks using IP (Internet protocol) as a common protocol so that users are in control. They suggested that $3 \mathrm{G}$ should be integrated with the IP based technology so that it can have tremendous data transmission and support VoIP as well.

Kaur and Kuma [18] carried out studies on efficient resource block allocation in LTE system. They aimed at using different scheduling algorithm in order to get the best throughput. They proposed algorithm that will first of all judge the scheduling block required by each user and after that assign scheduling block to each user according to their priority while allowing fair distribution of available resources among the users. Simulation results drawn from their study showed that there is an improvement in the overall system throughput.

A research was conducted on the main principles of the LTE network architecture by [20] They explained that LTE was designed to support only packet-switched services and aims to provide seamless Internet Protocol (IP) connectivity between user equipment (UE) and the packet data network (PDN), without any disruption to the end users' applications during mobility. They proposed that LTE is a better choice for next generation wireless mobile networks due to its simple architecture. They gave an overview of LTE and its architecture and the functions of both the core and access network. They also explained the Functional details and layout of the associated protocols. The objective of their re- 
search was to provide wireless mobile network with simple architecture and to give five categories of LTE interfaces namely Air interface, E-UTRAN interfaces, Core network interfaces, Mobility and interworking interfaces, and service interfaces. According to them, LTE Architecture is simple, flat IP based, reduces latency and cost, and it is compatible with 3GPP and non 3GPP Technologies. They emphasized LTE network element and Interface which is made up of UE, E-UTRAN and EPC.

Tantawy et al. [21] gave a study on cross layer scheduling algorithm. He explained that LTE came as aresult of an increasing need of next generation mobile networks to offer high performance, mobile broadband services. The objective of the work is to develop a cross-layer scheduling algorithm in LTE that will offer high performance, mobile broadband services, along with a combination of high bit-rates and system throughput in both the uplink and downlink along with low latency. A novel QoS guaranteed cross-layer scheduling algorithm for LTE system was proposed which allocates resources to the users as resource blocks.

Ayvazian [4] carried out a project on making 4G OFDM small-cell solutions smarter, scalable, cost effective and future proof. The thesis aimed at managing Interference in order to make $4 \mathrm{G}$ OFDM smaller, scalable, cost effective and future proof. The work uses an extensive portfolio of outdoor or indoor LTE small-cell solutions. Airspan invested in technologies for interference management in aggressive frequency reuse scenarios and offers a unique small cell with integrated non-line-of-sight and line-of-sight backhaul. They also developed a high performance, low cost solution for $3.5 \mathrm{GHz}$ operators that wants to deploy LTE Advanced Services using carrier aggregation.

\section{Proposed Model}

Evaluation of both networks were based on performance metrics like average throughput, total data rate, allocation of fairness, and latency of video streaming and VOIP traffic. Scheduling is allocating or reserving resources to users in a communication system to maximize throughput and system efficiency. A good Scheduling algorithm has two main goals: It gives higher spectral efficiency with fairness to all users and Fairness among users is balanced with throughput. Therefore, the overall goal of the scheduler is to satisfy the system and user requirement.

In this research, RR, best CQI and PF Algorithm were used majorly to compare the performance of $3 \mathrm{G}$ and $4 \mathrm{G}$ Network. A thorough evaluation was carried out on each factor that contributed to better resource scheduling using MATLAB simulator. MATLAB is ideal for the modeling and simulating the LTE network, as well as implement and test the algorithms on 3G and LTE network because, it has an in-built service called the LTE System Toolbox. This LTE System Toolbox accelerates LTE algorithm and physical layer development, supports conformance testing and test waveform generation [22]. The System performance metrics are based on:

1) UE's experienced total data-rate: is calculated using Shannon-Hartley 
theorem in Equation (1) [23].

$$
S_{i, k}(t)=x_{i, k}(t) \frac{B}{N} \log _{2} \log _{2}\left(1+\operatorname{SINR}_{i, k}(t)\right)
$$

where $B=$ the total bandwidth for the eNB.

$\mathrm{N}=$ the total number of RBs for the eNB.

$x_{i, k}(t)=$ the number of RBs allocated to user $i$ by $k$ at time slot $t$.

Note: One radio block (RB) has $180 \mathrm{kHz}$ bandwidth, 12 subcarriers, Time slot of $0.5 \mathrm{~ms}$ and 7 OFDM symbol

2) Average packet delay experienced by UE: delay is the difference in time between transmission from source and arrival at the destination. The average delay of the ith flow can be expressed by using Equation (2) [24]:

$$
D_{i}=\frac{1}{N} \sum_{j=0}^{N}\left[T_{d}(j) \mid-T_{s}(j)\right]
$$

where $T_{s}(j)$ s the time when the $j$ th packet was transmitted from its source, $T_{d}(j)$ is the time when the $j$ th packet was received at the receiver and $N$ is the number of packets used for calculating the average delay.

3) Average System throughput: this is measured as the total number of bits successfully transmitted over the air interface from the UE up to the eNodeB over the total simulation time as seen in Equation (3). That means the system average throughput is the sum of average throughput of all $4 \mathrm{G}$.

$$
\text { Throughput }=\frac{B}{t_{\text {sim }}}
$$

where $B=$ total amount of received bits $t_{\text {sim }}=$ total simulation time.

\section{Simulation Parameters for 3G and 4G Network}

The parameters for simulating both networks are shown in Table 2.

\subsection{Results and Analysis}

\subsubsection{Average System Throughput}

The results average throughput of $3 \mathrm{G}$ and $4 \mathrm{G}$ using different scheduling algorithms are as shown in Figure 3. 4G network has the highest throughput compared with that of 3G. Also, considering the different scheduling algorithm used, the graph shows that PFPS has the highest throughput followed by BestCQI while RR has the least throughput in $4 \mathrm{G}$ network.

\subsubsection{Data Rate}

In the entire Scheduling algorithm used, Data transmission rate is higher in $4 \mathrm{G}$ than $3 \mathrm{G}$ as shown in Figure 4. PFPS algorithm transmits data at a rate higher than BestCQI and RR in both 3G and 4G network. In 4G PFPS transmits data at about $60 \mathrm{Mbps}$, RR transmit at about $50 \mathrm{Mbps}$ and Best CQI at about $55 \mathrm{Mbps}$. With the result shown on the graph in Figure 6 we could conclude that PFPS gives the highest rate of transmitting data in $3 \mathrm{G}$ and $4 \mathrm{G}$ compared to other scheduling algorithms. 


\subsubsection{Latency}

The latency in $3 \mathrm{G}$ is higher for all the scheduling algorithms used, but $4 \mathrm{G}$ has a lower latency as shown in Figure 5. This will make connection and packet-transfer rate on a $4 \mathrm{G}$ network a lot faster than on a $3 \mathrm{G}$ network. It is also noticed that the PFPS algorithm seems to have the lowest latency among other schedulers; hence, it has a higher packet-transfer rate.

\subsubsection{Allocation Fairness}

Result shows that allocation fairness is almost the same in both $3 \mathrm{G}$ and $4 \mathrm{G}$ networks, for all the scheduling algorithms, except for PFPS as seen in Figure 6 . PFPS seems to be a good choice because it suggests an approach that is fair to the users and to the system resources, unlike Best CQI that takes channel quality into account before allocating resources to users and RR that wastes radio resources allocated to it.

Table 2. Simulation parameters for $3 \mathrm{G}$ and $4 \mathrm{G}$.

\begin{tabular}{ccc}
\hline PARAMETERS & VALUE (3G) & VALUE (4G) \\
Bandwidth & $1.4 \mathrm{MHz}, 3 \mathrm{MHz}, 5 \mathrm{MHz}$, & $1.4 \mathrm{MHz}, 3 \mathrm{MHz}, 5 \mathrm{MHz}$, \\
Access & $10 \mathrm{MHz}, 15 \mathrm{MHz}, 20 \mathrm{MHz}$ & $10 \mathrm{MHz}, 15 \mathrm{MHz}, 20 \mathrm{MHz}$ \\
Total users & WCDMA & LTE \\
Number of RBs & 15 & 15 \\
Number of Sub frames & Ceil (numRBs/2) & Ceil (numRBs/2) \\
RT percentage & $40 \%$ & $40 \%$ \\
UR percentage & $30 \%$ & $30 \%$ \\
NRT percentage & $30 \%$ & $30 \%$ \\
Scheduling Algorithm & PFPS, Best CQI, Round Robin & PFPS, Best CQI, Round Robin \\
\hline
\end{tabular}

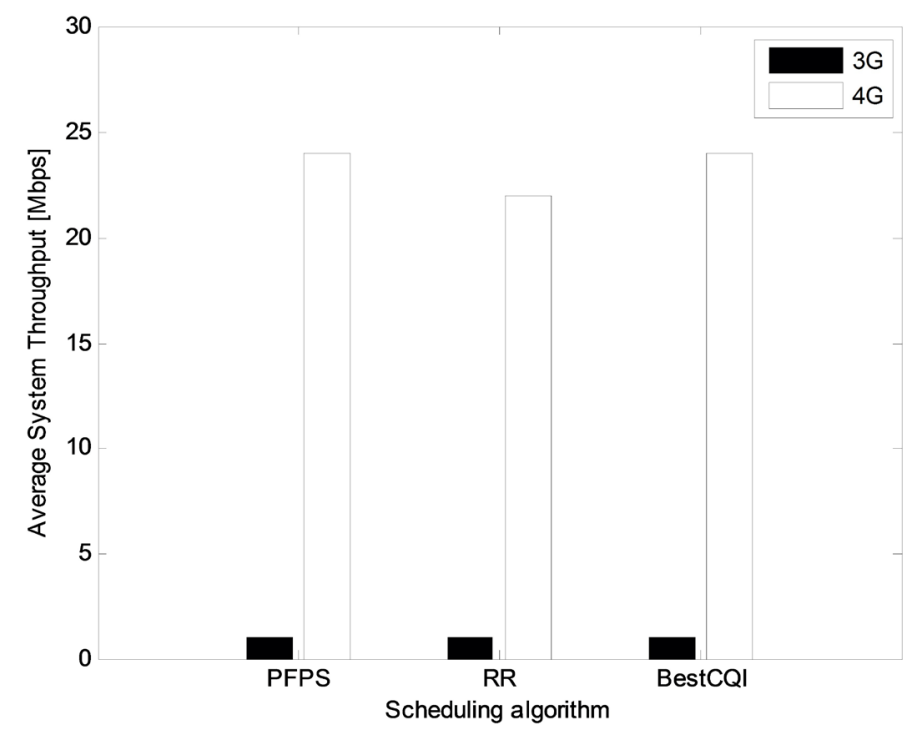

Figure 3. Average system throughput (Mbps). 


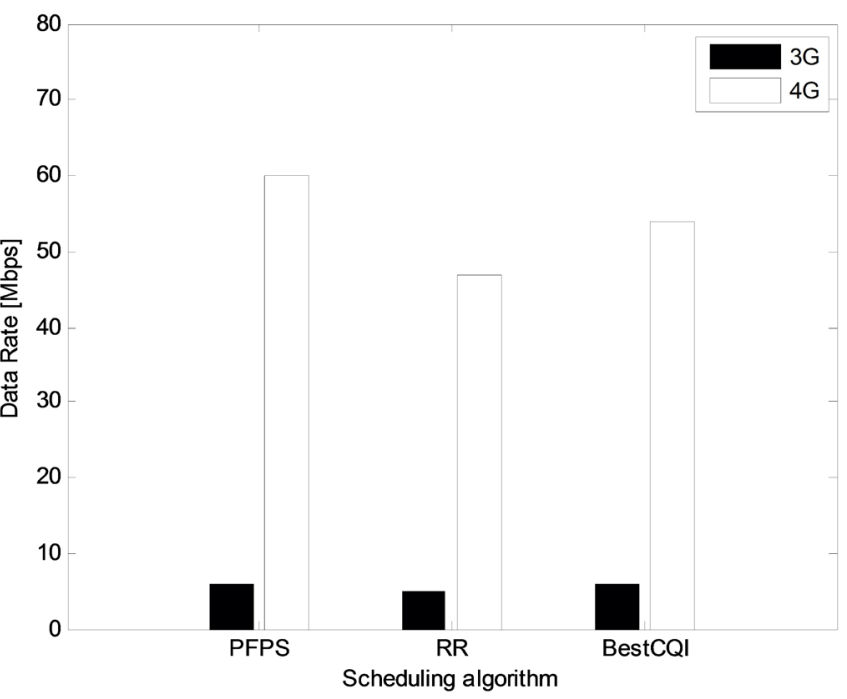

Figure 4. Data Rate (Mbps).

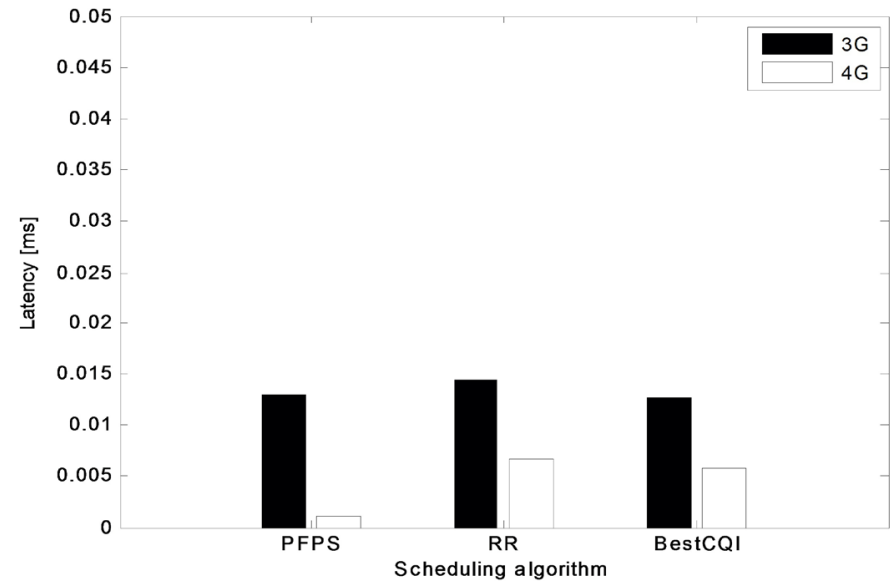

Figure 5. Latency (ms).

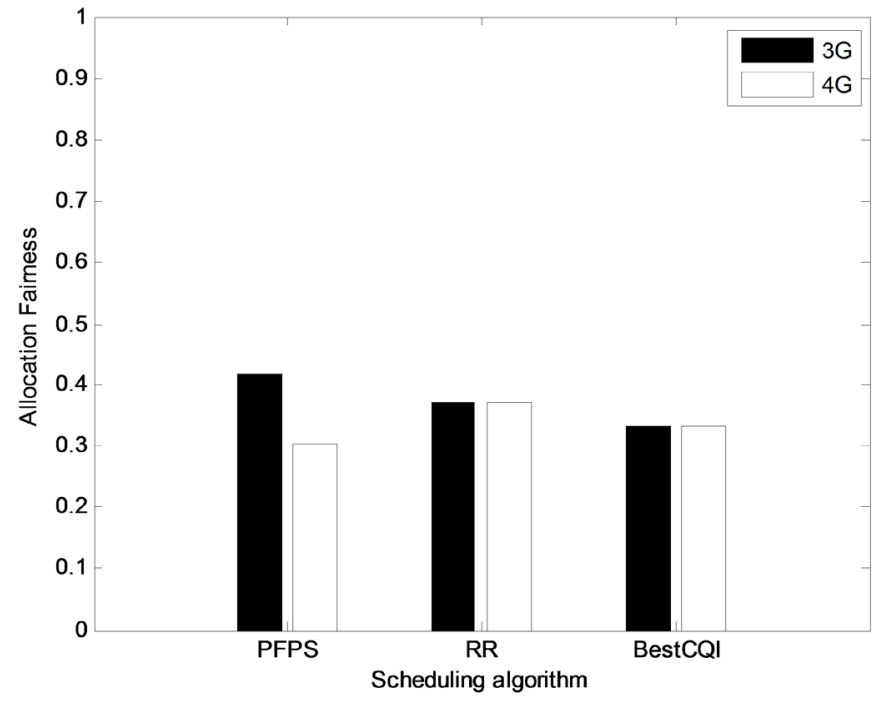

Figure 6. Allocation fairness. 


\section{Conclusion}

The comparative analysis carried out on $3 \mathrm{G}$ and $4 \mathrm{G}$ Network with different scheduling algorithms, showed that $3 \mathrm{G}$ performance is still very low in terms of meeting the major requirement of a quality network. Results from the work show that $4 \mathrm{G}$ network is far better than $3 \mathrm{G}$ in provision of quality services that enables users have access to the network at a very high data speed, high bandwidth and low latency. Based on the fact that LTE network provides a high speed of data transmission, access to quality internet services such as content downloading, video streaming, quality voice communication; Government and Cellular operators should try as much as possible to allow the deployment of LTE network in Nigeria so as to improve the user's experience when accessing the Internet.

\section{Conflicts of Interest}

The author declares no conflicts of interest regarding the publication of this paper.

\section{References}

[1] Mojtahed, M. and Xirasagar, S. (2013) Quality of Service over LTE Networks. White Paper.

https://www.wirelessdesignmag.com/article/2013/09/quality-service-over-lte-netwo rks-part-1-3

[2] UN News.

https://news.un.org/en/story/2017/07/562562-mobile-broadband-subscriptions-trac k-hit-43-billion-2017-un-report

[3] https://www.statista.com/statistics/206615/forecast-of-the-number-of-global-hspa-lt e-subscriptions-up-to-2014/

[4] Ayvazian, B. (2013) LTE TDD Services in the 3.5GHZ Bands. Opportunities and Challenges for LTE, Heavy Reading, Special Research Report.

[5] Sharma, P. (2013) Evolution of Mobile Wireless Communication. International Journal of Computer Science and Mobile Computing, 2, 47-53.

[6] Kuboye, B.M. and Alese B.K. (2009) The Pathway of GSM to 3G Systems in Nigeria. Pacific Journal of Science and Technology, 10, 388-397.

[7] Kuboye, B.M. (2014) Mobile Communication Evolution. International Journal Modern Education and Computer Science, 6, 25-33. http://www.mecs-press.org/

[8] Ozovehe, A. and Usman, A.U. (2015) Performance Analysis of GSM Networks in Minna Metropolis of Nigeria. Nigerian Journal of Technology, 34, 359-367. https://doi.org/10.4314/njt.v34i2.21

[9] Rouffet, et al. (2005) 4G Mobile. Alcatel Telecommunications Review. http://www.alcatel.com/atr

[10] Jalier, et al. (2010) Heterogeneous vs Homogeneous MPSoC Approaches for a Mobile LTE Modem. Date: Design, Automation and Test in Europe, Dresden, March 2010, 184-189. http://www.date-conference.com/

[11] Adokar, D.U. and Rajput, P.J. (2012) Wireless Evolution with 4G Technologies. International Journal of Advanced Research in Electrical, Electronics and Instrumen- 
tation Engineering, 1, 323-333.

[12] Kirilov, Z.N. (2011) Optimized Service Aware Radio Resource Scheduler in LTE. Master's Thesis, Department of Communication Networks, University of Bremen, Many.

[13] Rakesh, K.S. and Ranjan, S. (2016) 4G LTE Cellular Technology: Network Architecture and Mobile Standards. International Journal of Emerging Research in Management \& Technology, 5, 1-6.

[14] Sukar, M.A. and Pa, L. (2014) MSC-FDMA and OFDMA in LTE Physical Layer. International Journal of Engineering Trends and Technology, 12, 74-85.

[15] Ibrahim, K. and Badreldin, E.B. (2013) Long Term Evolution and Optimization Based Downlink Scheduling. International Journal of Computer Application, 71, 13-23.

[16] (2009) LTE Resource Guide. http://web.cecs.pdx.edu/ fli/class/LTE_Reource_Guide.pdf

[17] Anjorin, O. and Arslan, Ö. (2011) Performance Evaluation of Packet Scheduling Algorithms for LTE Downlink. Blekinge Institute of Technology, Master of Science in Electrical Engineering, Sweden.

[18] Kaur, R. and Kumar, M. (2013) An Efficient Resource Block Allocation in LTE. International Journal of Advance Research in Computer Science and Software Engineering, 10, 1151-1156.

[19] Kumar and Suman (2013) Comparision of 3G Wireless Networks and 4G. International Journal of Electronics and Communication Engineering, 3, 1-8.

[20] LTE Network Structure: A Comprehensive Tutorial, Alcatel-Lucent White Paper SudeepPalat and Philippe Godin Lead representatives in Alcatel-Lucent's 3GPP Standardization Team (2010).

[21] Tantawy, M.M., Eldien, A.S.T. and ZakiR, M. (2011) Cross Layer Scheduling Algorithm for Long Term Evolution. Canadian Journal on Multimedia and Wireless Networks, 2, 57-62.

[22] MathWorks. Simulink Documentation. http://www.mathworks.com/help/

[23] Rioul, O. and Magossi, J.C. (2014) On Shannon's Formula and Hartley's Rule: Beyond the Mathematical Coincidence. Entropy, 16, 4892-4910.

[24] Borve, H.B. (2008) Packet Scheduling Algorithm for Wireless Network. Master of Science in Communication Technology, Norwegian University of Science and Technology, Trondheim. 